\title{
A Case-Study on the Effect of Averaging Duration on RF Dosimetry of General Public Environments
}

\author{
Reza Asadi \\ Electrcial Engineeing \\ Faculty, K.N. Toosi \\ University of Tehnology \\ Tehran, Iran \\ $\underline{\mathrm{R} \text { asadi@email.kntu.ac.ir }}$
}

\author{
Piroz majdolashrafi \\ Electrcial Engineeing \\ Faculty, K.N. Toosi \\ University of Tehnology \\ Tehran, Iran \\ pmajd@mail.kntu.ac.ir
}

\author{
Ghazale Khayambashi \\ Electrcial Engineeing \\ Faculty, KN Toosi \\ University of Tehnology \\ Tehran, Iran \\ khayambashi@email.kntu.ac.ir
}

\author{
Hadi Aliakbarian \\ Electrcial Engineeing \\ Faculty, KN Toosi \\ University of Tehnology \\ Tehran, Iran \\ h.aliakbarian@ieee.org
}

\begin{abstract}
Electromagnetic waves can cause undesirable effects on human body. These hazards have forced the related certification bodies such as ICNIRP and IEEE to propose standard limitations on the electromagnetic field levels. However, the current recommended procedures demand to verify the safety compliance of each measurement point based on a lengthy and impractical 6-minute averaging, in addition to spatial averaging, which complicates the procedure of $\mathrm{RF}$ dosimetry. Our statistical analysis on the performed experimental indoor and outdoor measurements show that lower time interval of measurements, such as 30 seconds and 60 seconds, predicts the same results in common electromagnetic environments, affected by wireless networks and broadcasting signals. The overall observed difference between 30 -second and 6-mintue averaging time length from our reference value is less than $50 \%$ respectively in $13.5 \%$ and $10 \%$ of times, illustrating that there is not a significant difference between 30 -seconds and 6-minute averaging. This makes the RF dosimetry procedure much faster, and cheaper while keeping it almost as reliable as before.
\end{abstract}

Keywords-ICNIRP, RF dosimetry, general public, specific absorption rate, electromagnetic waves

\section{INTRODUCTION}

Nowadays with fast spreading use of electromagnetic systems, such as handheld devices, IoT-based home appliances, and even high-tech automobiles, no one can relinquish the concern of RF waves influence on human body and health. Studies show RF waves might cause fever-like high body temperature. There are reports that it plays role in reproductive system and Central nervous system disorders[1]. In the last decade, this issue has become a source of worry to health care societies. Therefore, many researches have been carried out on a wide range of people to shed light on their influences on different diseases. For example, in [2, 3] the risk of developing acoustic neuroma by using cellular phone is investigated. In [4], the effect of non-ionizing radiation on children has been studied. and the effects of using mobile phones have examined by [5-7], and. In [8], the effects of Wi$\mathrm{Fi}$ waves on the pregnant mother's health have been investigated. Also in [9], the effects of mobile waves on sleeping quality of 18 to 19 years old people are studied. In [10], the human hazards of electric cars' wireless chargers at $5.6 \mathrm{GHz}$ on human eye cells has been investigated. No statistically significant effect on the DNA of the cells are shown as a result of exposure in this paper.

Various researches on the measurement of electromagnetic wave levels in public urban and rural areas has been conducted in the recent years. As stated in [11], the measurement process of these works are different. Measurements are made by fixed measurement stations, portable dosimeter devices such as spectrum and antenna, or by using personal dosimeters connected to the person's body which constantly monitors radiation in each locations.

Various studies have been reported on different public environments by using one of the above mentioned methods. Jalilian et al [12] has investigated the effect of the increase of using wireless devices over several years in urban environments, especially in public places, in difference urban cities and compared the results with those of three years before. Although the amount of services has been changed during these years, the measured level has not been change significantly. In [13] the radiated waves level in several offices are considered in $80 \mathrm{MHz}$ to $6 \mathrm{GHz}$. The results show that the average power density is $0.524 \mathrm{~mW} / \mathrm{m}^{2}$ for weekdays and $828 \mathrm{~mW} / \mathrm{m}^{2}$ for weekends which shows an increased level in the weekends. Rachel Nkem Iyare et al [14] have measured the levels of signals by using a spectrum analyzer and a portable antenna in GSM 900 , GSM 1800 and UMTS at 60 points Leuven, Belgium. All values measured in this article are significantly lower than the ICNIRP exposure limit for the general public. On average, the outdoor exposure factor is twice as high as the indoor level. The average is reported for the external environment of $0.5 \mathrm{v} / \mathrm{m}$ and $0.153 \mathrm{v} / \mathrm{m}$ for the indoor environment. and then compared their results with other similar studies.

Due to these concerns, there are specialized groups working on the effects of these waves. The outcome of these studies have resulted in the proposition of a comprehensive standard that provides rules and restrictions on the basis of scientific experiments and researches for protecting against potential dangers of electromagnetic waves. These standards include international IEEE C95 [15] and ICNIRP [16] and national standards such as Australian Standard, and Canada Health Document No. 6 [17], and the national standard in Iran, INSO-8567 .

According to these standards, the proper time interval for EM measurements regardless of the environment is 6 minutes. Foster [17] has noted that he himself has proposed this averaging time length (ATL), to be one tenth of an hour (0.1 h), or 6 minutes, so that not to be long and no to be short. This value has been verified by the providers of the guidelines and has not been changed till now. This long measurement time interval is the main problem that we address in this paper. The origin of this averaging time length is the thermal effects of the duration of exposure on the body. However, the main objective of this paper is not to investigate this effect but to study the averaging time needed to have a reasonable estimation of the electric field value which cause this effect. Of course, the longer the averaging is performed, the more reliable electric field value for each measurement point is obtained. However, we will show that at least in certain 
conditions, this averaging time length (ATL) can actually be shorter. The required ATL can be different based on the specifications of the transmitters around. In this study we assume that the environment is a normal environment which is only exposed to normal RF sources such as TV, radio, cellular and WLAN networks signal.

In this paper, national and international standards as the main reference guidelines are discussed in section II and the experimental setup which is used in our studies is introduced. In section III, the obtained experimental results are demonstrated. In this section, the effect of averaging time length on measuring performance considering threshold levels in accordance with ICNIRP and national Iranian standards has been investigated. The results of these local measurement studies are numerically analyzed. As a result, we have shown that lowering the measurement time intervals does not significantly affect the accuracy of the measurement standards

\section{RF DOSIMETERY STANDARDS}

\section{A. Introduction and comparision among standards}

International Commission on Non-Ionizing Radiation Protection (ICNIRP) as the most reliable organization presenting international standards against high electromagnetic radiation was founded in 1974. This organization was formed from an international radiation protection association against IRPA with the help of World Health Organization (WHO), in order to regularize electromagnetic waves level in different environments[16]. These proposed limits have been summarized based on the amount of the tolerable Specific Absorption Rate (SAR). $\mathrm{SAR}$ is an index which measures electromagnetic energy absorbed by biological tissues and turned into heat. The proposed limit in this documents are determined by considering the direct and indirect effects of electromagnetic waves on human body, experimental results and epidemiologic studies between $1 \mathrm{~Hz}$ to $300 \mathrm{GHz}$ range of frequencies (Figure 1), which are concluded according to special demographic groups, such as weak, sick, elder, children and infants. These standards categorize their limitation levels into two levels, one for controlled and the other one for non-controlled environments, which are explained according to complementary information in[16]. These standard levels are the best available criteria to the scientific community for evaluating possible electromagnetic dangers.

IEEE C95 code as another reliable international recommendation has been presented and developed by international electromagnetic safety committee[18]. IEEE recommendation is rather similar to ICNIRP's but it starts from $3 \mathrm{kHz}$ up to $300 \mathrm{GHz}$ frequency (Figure 1). The recommendations are based on extensive studies of researchers and experts in this field. Some countries have published their own standard for protecting people against harmful effects of electromagnetic waves. Canada's Health organization has paid attention to the effects of these waves by forming a group, and finally published its recommenddation based on scientific studies in 1999 with the name of document No.6 [17](Figure 1).

Finally, the standards are divided into both public environment, such as residential areas, schools and urban streets versus non-controlled categories such as transmit/receive centers of TV communication waves. In
Figure 1, a comparison has been done according to the last published limitation levels of these standards, showing a similar frequency dependent behavior in all of them.

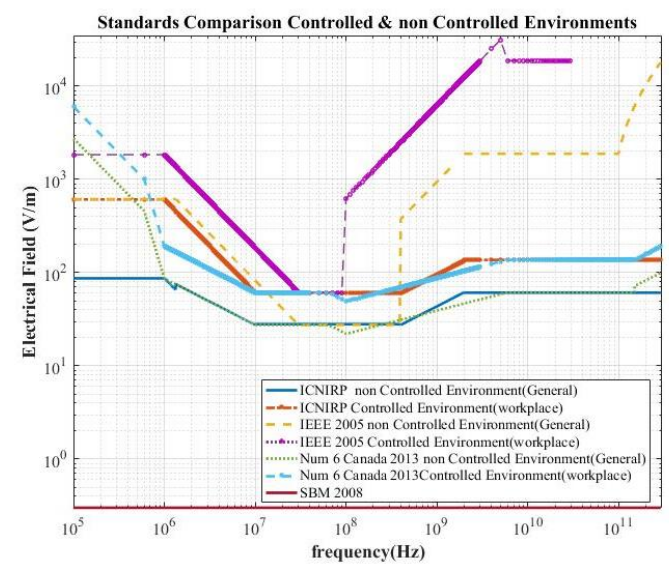

Fig. 1. Comparison among limit levels of four standards.

\section{B. Measurement procedure based on standards}

In order to verify safety of an environment or location from electromagnetic waves point of view, some practical procedures have been recommended by the standards. The measurement procedure, which has been used in our work, is based on the procedures introduced in $[16,17]$. Due to its popularity, ICNIRIP standard, shown in Figure 1, is chosen as a benchmark standard. This commission has a reasonable mechanism of making decisions based on scientific researches. In addition, its international standard is utilized by many national organizations.

In the next step, the environmental conditions should be determined, whether it belongs to public (uncontrolled) environments or work (controlled) environments.

- Work environments (controlled): is said to an environment in which the present people has enough information about waves and their presence time is less than 8 hours per day.

- Public environment (uncontrolled): is said to an environment in which the present people have no information about waves and their presence time is less than 24 hours per day, such as home, school, restaurant, etc.

In the third step, the measurement devices are set up in the place. This device should be able to measure waves from different directions without changing their characteristics. Spectrum analyzers plus an antenna and field measurement probes are two examples of this measurement device.

In the fourth step, the measurement starts in an environment which is in its normal stable conditions. It should be noticed that recommended measurement time for each point takes 6 minutes long based on all standards. Average time of results are calculated by using Equations (1 and 2) from Canada safety code No.6 [17]

$$
\begin{gathered}
W_{j}=\frac{1}{T} \sum_{i=T_{o}}^{T+T o} W_{i j} \\
W_{\text {average }}=\frac{1}{m} \sum_{\mathrm{j}=1}^{m} W_{j}
\end{gathered}
$$


In equation (1), $W_{j i}$ is the $i^{\text {st }}$ measured power level sample, $\Delta t_{i}$ is the time difference between two samples, $t_{i}$ and $t_{i-1}$, and then averaging is performed over all $n$ samples in $T=6$ minutes. $W_{j}$ is the time averaged power in the $j^{\text {th }}$ measurement sub-position which is also used in equation (2) where it is spatially averaged over all $m$ measurement sub-positions to obtain $W_{\text {average }}$ as the power value of a specific position. All of the above-mentioned $m$ sub-positions are performed on a flat plane with an approximate size of an adult person. The averages of measurements, namely $W$, are finally compared with the determined limits of the standard.

\section{MEASUREMENT}

\section{A. Purpose of Measurement}

As stated in the previous section, the RF measurement averaging at each point should be done for 6 minutes at both public and workplace environments[16].

Given the fact that the wave level varies in different environments, each of these two environments can be divided into several sub-categories. The public environment can be divided into several subcategories depending on the propagation channel model and the source of electromagnetic waves in the environment, , for instance, academic environments such as universities and schools, large shopping malls, office apartments, residential apartments, and small suburban homes.

This paper aims at investigating the feasibility of reducing the $\mathrm{RF}$ averaging time in some specific environments in order to reduce the time and the cost of dosimetry and makes it affordable for normal customers. The main feature of this environment is being located in the public wave propagation complex, which includes mobile radio, TV transmitters and Wi-Fi. In this environment, there is no cross-sectional activity to increase the wave level in the environment.

\section{B. Measurement Setup}

In order to verify the above mentioned study case, a test procedure has been designed, based on the use of NBM 550NARDA. It uses EF1891 directional probe which is able to measure electric field in $3 \mathrm{MHz}$ to $18 \mathrm{GHz}$ with $1 \mathrm{uV} / \mathrm{m}$ accuracy in three polarizations. Its processing power allows us to perform RF dosimetry sampling with the frequency up to $1 \mathrm{~Hz}$ and measuring up to several days continuously over time. In all of these environments, the probe height is $1.7 \mathrm{~m}$ above floor and measurement times is 2-8.5 hours. During this measurement, the average of received power in environment is recorded in $3-18000 \mathrm{MHz}$ frequency range. Figure (2) shows measurement setup installed in the environments.
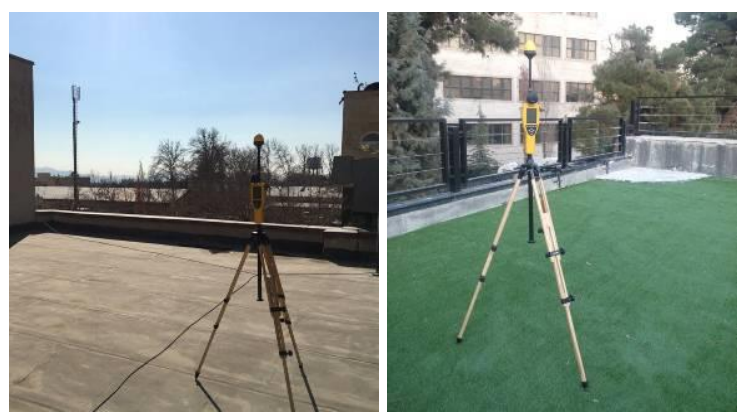

Fig. 2. Measrurement instruments in two of the locations.

\section{Measurment procedure}

The specific environment of this study is a university academic environment. The existing waves in the environments would widely vary throughout the day. Experiments are conducted at K. N. Toosi University of Technology Electrical Engineering campus in downtown Tehran, Iran. This place is categorized as a public environment defined in the standard. The measurements are carried out in several buildings and locations including two indoor environments (an office - a research laboratory and a computer center) and three outdoor areas including a cafe - a sports ground and a library building terrace.

Each test is performed for 2 to 9 hours under normal conditions over a period of 6 months. During each test, the device stores 1 sample power density every 1 second. Overally, during this process, more than 100,000 samples have been gathered. The probe's condition and position in the environment are adjusted so that no transmitter is in close vicinity. The height of the probe is approximately 150 to $170 \mathrm{~cm}$ due to average height of a human body while standing. The test has examined the normal conditions of a person living or working in such environments and having no knowledge of the emitted waves. Figure 2 shows the measurement setup.

\section{RESUlTS AND DISCUSSIONS}

\section{A. Scenraio}

In this scenario, it is assumed that the operator sets up the device to measure RF field level at a point. The measurement start time can be any moment during day or night. Since the purpose of the paper is to investigate the possibility of reducing the averaging time length (ATL), the question is how does it affect the measured RF level results. Therefore, the test data have been captured for a much longer times than the standards with 1 second resolution. For instance, our two-hour recording has 7200 samples per location. Now, each of these 7200 seconds, let say $T_{o}$, can be set as the Start Moment of Averaging (SMoA) and thus the averaging is performed from the $\mathrm{SMoA}$, or $T_{o}$ in Equation (1), to $T_{o}+T$ thereafter and then is averaged using Equation (2) for the ATL seconds, namely $T$ seconds in Equation (1). It then completes the assumption under discussion to examine the effect of ATL (or $T)$ and SMoA $\left(T_{o}\right)$.

\section{B. Effect of $A T L$}

The effect of three different ATL values, $T=1$ min, $T=0.5 \mathrm{~min}$, and $T=6 \mathrm{~min}$ are investigated by comparing them when sweeping SMoA, shown in Figures 3 and 4.

The compared factor is the Average Power Density (APD) of each of these three cases, which is calculated by using Equation (1). The measurements are compared in three locations, the sport ground, a café and the central library.

The horizontal axis of these figures shows the SMoA which is the time of starting to average, $\left(T_{o}\right.$ in Equation (1)) in seconds. 
As can be seen in Figures 3(a) to 3(c), measured in outdoor environments, APD (Averaged power density) has variations in all three averaging periods (30 seconds 3 minutes and 6 minutes) during these 2 hours due to time varying specifications of the signals, However, it is visually clear that for identical SMoAs $\left(T_{o}\right)$, there are slight differences among the three cases. In more general view, for all of 100,000 longterm test samples performed in our experiments the percentage of APD difference between the samples of 30-second APD and 6-minute APD is nearly always less than $3 \mathrm{~dB}$ as will be discussed later. Figures 4(a) to 4(c) also demonstrates the same measurement but for three indoor environments, two laboratories and

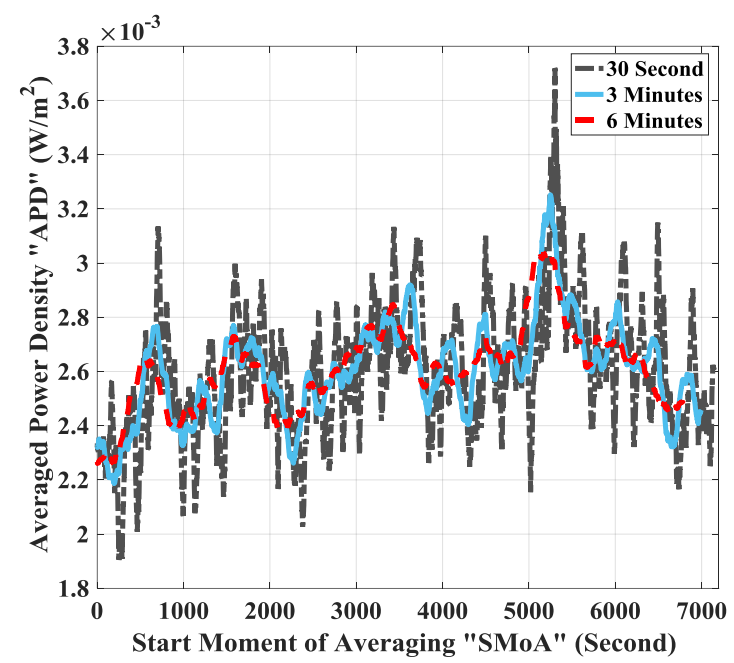

Sports ground (a) one office. Again, the results visually show the APD comparison among 30 seconds, 3 minutes, and 6 minutes ATL saying that although the variations are less in 6 minutes ATL, but the difference between all three cases is not great and can be ignored in many cases. Figure 5 plots the APD range changes measured for an environment based on different ATLs which shows that average APD value does not change drastically by ATL, although the maximum APD values gradually decreases as ATL increases. It can also be seen that the frequency of values near average value is much higher than value near maximum values.

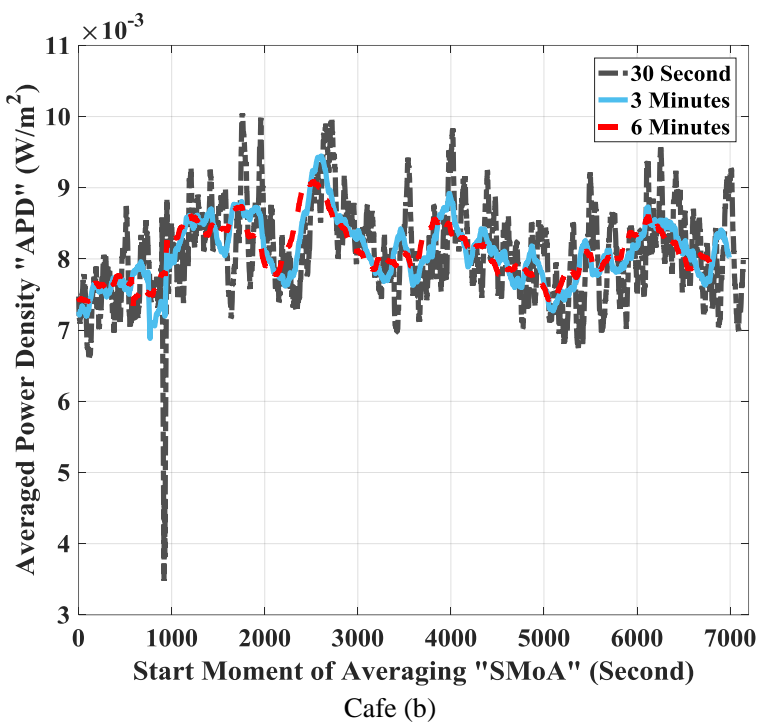

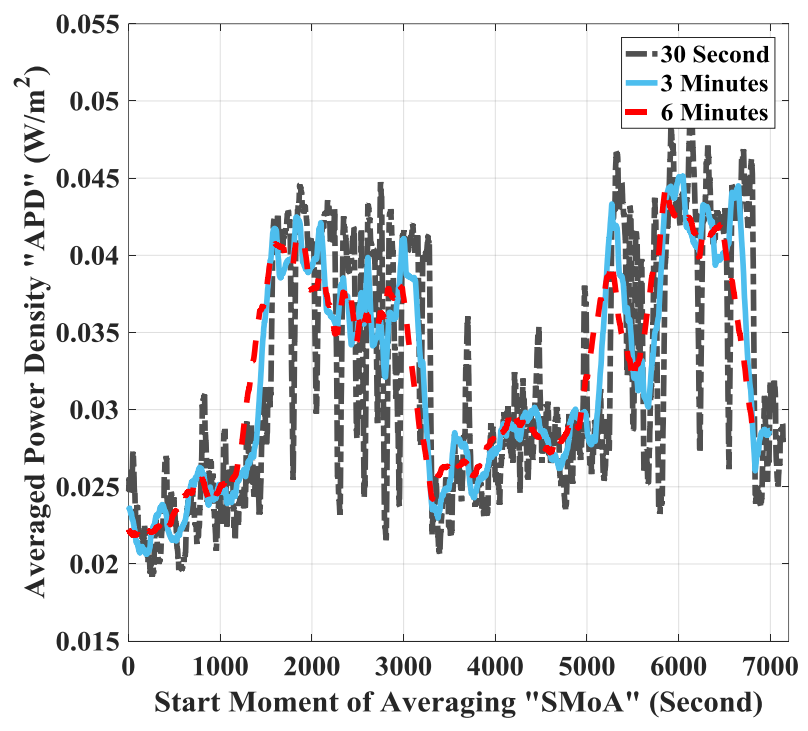

Central library terrace (c)

Fig. 3. A comparison among three ATLs started to over time and for three outdoor environments. Each value in the plot shows the APD (Averaged power density) value which is averaged from that moment (SMoA) and ends 30 seconds, 3 minutes, and 6 minutes later respectively. 

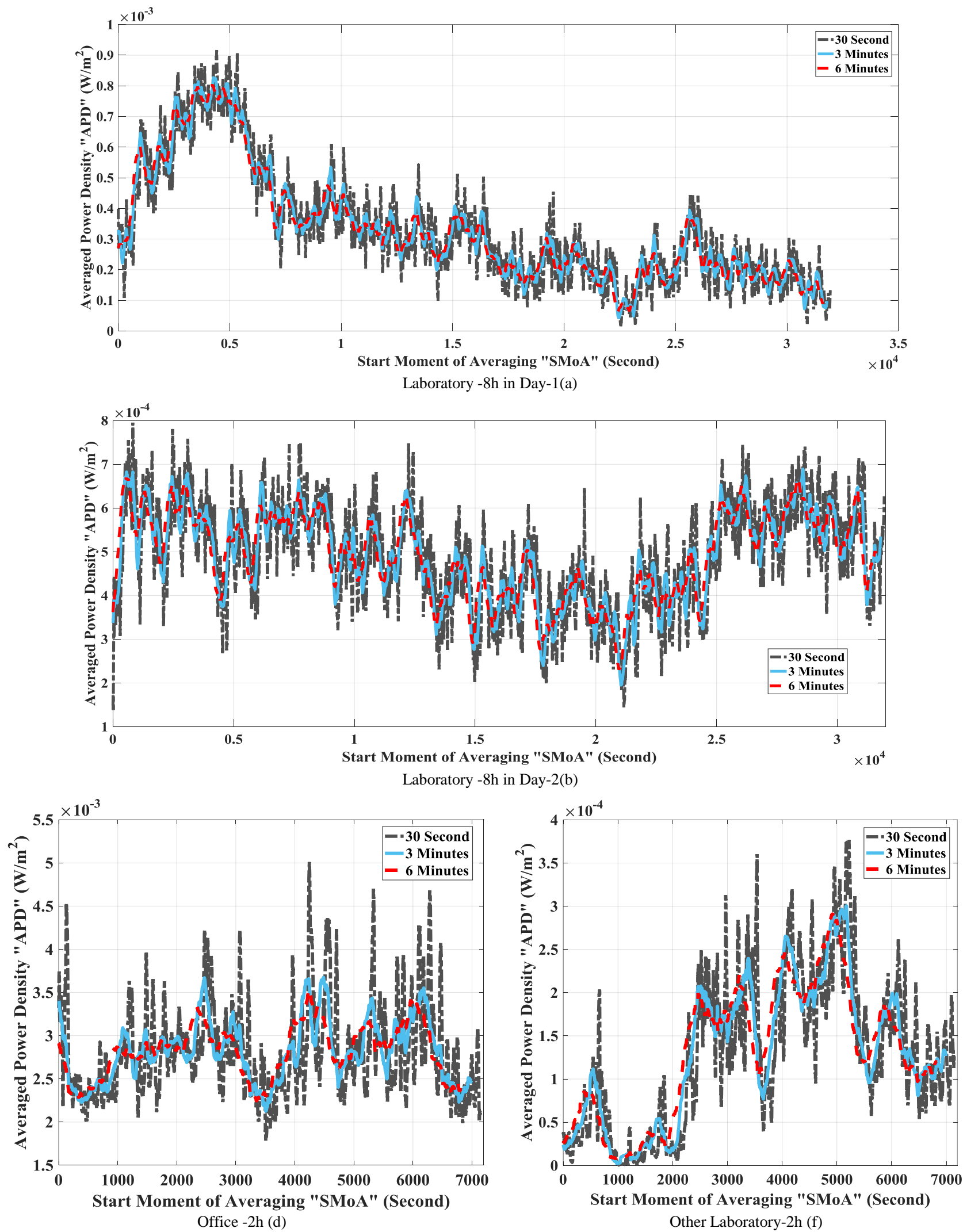

Fig. 4. APD comparison among three ATLs (30 seconds, 3 minutes, and 6 minutes) plotted versus SMoA (up to 7000 seconds) and for three indoor environments. Each value in the plot shows the APD (Averaged power density )value which averaged from that moment (SMoA) and ends 30 seconds, 3 minutes, and 6 minutes later 


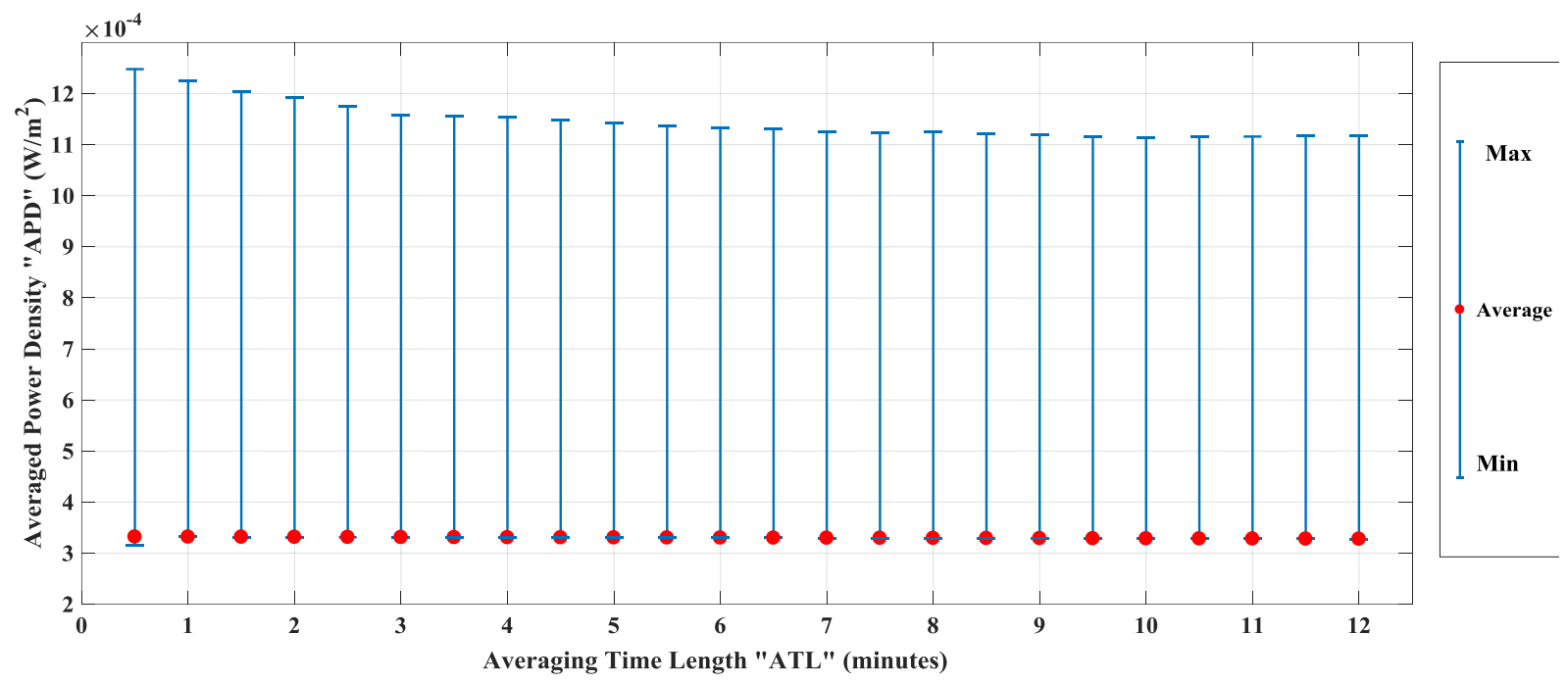

Fig. 5. Range of changes of samples measured at different measurement times (30 seconds to 12 minutes)

\section{Theoretical Discussion}

An array of $M$ measured power density samples in the environment, called $x[n]$, can be expressed in terms of the actual value of the average, namely $x_{0}$ and the error value $W[n]$, shown in Equation 4a. The actual value, $x_{0}$, is not available to us. However, Equation $4 \mathrm{~b}$ is statistically used to estimate it from $N$ captured $x[n]$ samples.

$$
\begin{aligned}
& X[n]=x_{0}+W[n] \\
& \hat{x}_{0_{s}}^{N}=\frac{1}{N} \sum_{i=s}^{S+N} X[n]
\end{aligned}
$$

where $\hat{x}_{0_{s}}^{N}$ is the estimated value from $N$ number of samples started from the $s^{\text {th }}$ samples. The variance of the difference between the estimated value and the actual value can be defined and calculated from Equation $5 \mathrm{a}$ and $5 \mathrm{~b}$

$$
\begin{gathered}
\sigma_{\hat{x}_{0}^{N}}^{2}=\frac{1}{M-N} \sum_{s=1}^{M-N} \sigma_{s}^{2} \\
\sigma_{s}=\left|\hat{x}_{0_{s}}^{N}-\hat{x}_{0}^{M}\right|
\end{gathered}
$$

where $\widehat{x}_{0}^{M}$ is estimated by averaging from the largest number of available samples, say $M=32000$ for 8 hours, while $N$ can be selected as a smaller number of samples, say 30,60 or 360 (6 minutes). $\hat{x}_{0}^{M}$ does not depend on $s$ and is the best estimation of the actual value $x_{0}$ [19].

If we plot the variance of the differences $\sigma_{\hat{x}_{0}^{N}}^{2}$, versus any length of samples, $N$, it is observed that as $N$ becomes larger, $\sigma_{\hat{x}_{0}^{N}}^{2}$ converges to 0 as $\hat{x}_{0_{s}}^{N}$ approach $\hat{x}_{0}^{M}$. Figure 8 and 9 shows this plot for both cases, indoor and outdoor. From the 8-hour indoor plot of Figure 8 , it is observed that only 11 seconds is enough until $\sigma_{\hat{x}_{0}^{N}}^{2}$ reaches below out threshold of $10 \%$ error, defined as one tens of $\hat{x}_{0}^{M}$. This value becomes 20 seconds and 80 seconds for 5\% and $1 \%$ errors respectively. Figure 9 shows the results of a 2-hour measurement in an outdoor environment. These results show that in the sample length, $N$, needed to reach $10 \%, 5 \%$ and $1 \%$ errors are 4 seconds, 6 second, and 19 seconds respectively which is much better than indoor test due to its fewer multipath.

Here, the lengths of each samples, $N$ is equal to Averaging Time Length, ATL.

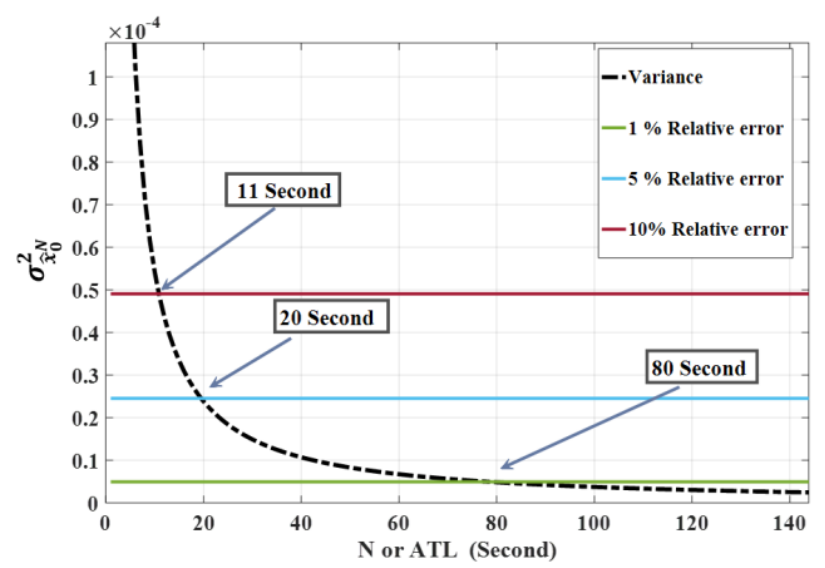

Fig. 6. Variance of the difference for $N$ seconds meausmrenrs from 8hour average, $\sigma_{\hat{x}_{0}^{N}}^{2}$ for an indoor example. 


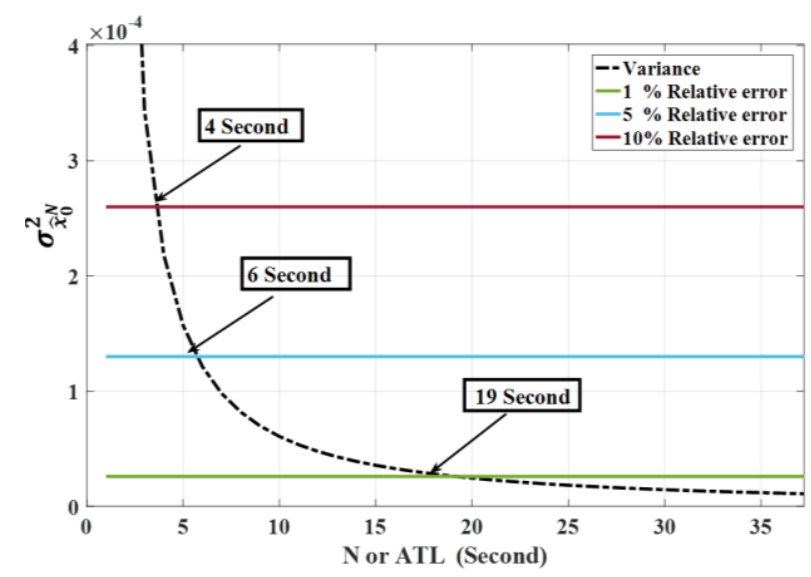

Fig. 7. Variance of the difference for $\mathrm{N}$ seconds meausmrenrs from 2 hour average, $\sigma_{\hat{x}_{0}^{N}}^{2}$ for an outdoor example.

\section{Comparing 30-second and 6-minute ATL}

In order to compare two ATL candidates, 30 seconds and 6 minutes, the best approximation, namely $M$ sample averages (8-hour and 2-hour) are selected. A new variable called "Relative Diversion from M-sample Average Power Density", or "RD from M APD” in brief, is defined in Equation (3).

$$
\text { “RD from } M \text { APD” }=\left[\frac{\left|A P D_{A T L}(T o)-A P D_{M}(T o)\right|}{A P D_{M}(T o)}\right] \times 100
$$

$$
A T L \in\{30 \mathrm{~s}, 6 \mathrm{~min}\}
$$

where $M$ is the total sample length measured in the medium, here $M=8000$ and 32000 , and $t$ is the SMoA for calculating APD.

Figures 8 (a, b, and c) show the "Relative Diversion from 2-hour APD" for two other ATL values, namely 30 seconds and 6 minutes, compared for outdoor environments. Figure 9 $(\mathrm{a}, \mathrm{b}$, and $\mathrm{c})$ do the same for indoor environments. The difference, shown in $\mathrm{dB}$, in both indoor and outdoor locations, demonstrates that decreasing ATL from 6 minutes to 30 seconds do not significantly affect the similarity of their APD to the reference level, which is the best approximation APD. Table 1 compares the "RD from 2-hour APD" of 30second measurements with its 6-minute rival. In each column of the table, the percentage of the time in which relative diversion from the reference value is above $10 \%$ and $50 \%$ threshold level is reported. In most cases, the results of both 6-minute and 30-second case are very close. However, it is only in the second row out of all seven rows that the amount of $10 \%$ difference is significant. In the last row of the table, the results are averaged showing that, in $52 \%$ and $62 \%$ of the time, the RD is less than $10 \%$ respectively for 30 -second and 6-minute measurements. The results are $10 \%$ and $13.5 \%$ respectively for $50 \%$ error of 30 -second and 6-minute measurements.

The 8-hour long calculation of " $\mathrm{RD}$ from 6-mintue APD" for the laboratory, which is shown in Figure 9 (a) follows the same results as the results of indoor calculation in Figure 7.
Table 1 Percentage of time in which the difference of 30 -second

\begin{tabular}{|c|c|c|c|c|}
\hline & \multicolumn{2}{|c|}{$\begin{array}{l}A P D \text { " is less than } 10 \\
\text { (\%) of time when RD is less } \\
\text { than } 10 \%\end{array}$} & \multicolumn{2}{|c|}{$\begin{array}{c}\text { and } 50 \% \\
\text { than } 50 \%\end{array}$} \\
\hline & For 6-min & For 30-sec & For 6-min & For 30-sec \\
\hline $\begin{array}{c}8 \text { hour- } \\
\text { Day1 }\end{array}$ & 94.48 & 90.40 & 43.32 & $\begin{array}{l}54.21 \\
\end{array}$ \\
\hline $\begin{array}{l}8 \text { hour- } \\
\text { Day2 }\end{array}$ & 29.10 & 59.20 & 0 & 1.99 \\
\hline Indoor-1 & 80.35 & 84.70 & 26.74 & 35.70 \\
\hline Indoor-2 & 73.99 & 71.44 & 0 & 2.19 \\
\hline Outdoor-1 & 5.66 & 26.36 & 0 & 0 \\
\hline Outdoor-2 & 0 & 19.97 & 0 & 0.056 \\
\hline Outdoor-3 & 79.04 & 83.56 & 0 & 0.55 \\
\hline Average & 51.80 & 62.23 & 10.01 & 13.52 \\
\hline
\end{tabular}
and 6-minute measurement from the reference value, " $R D$ from $M$
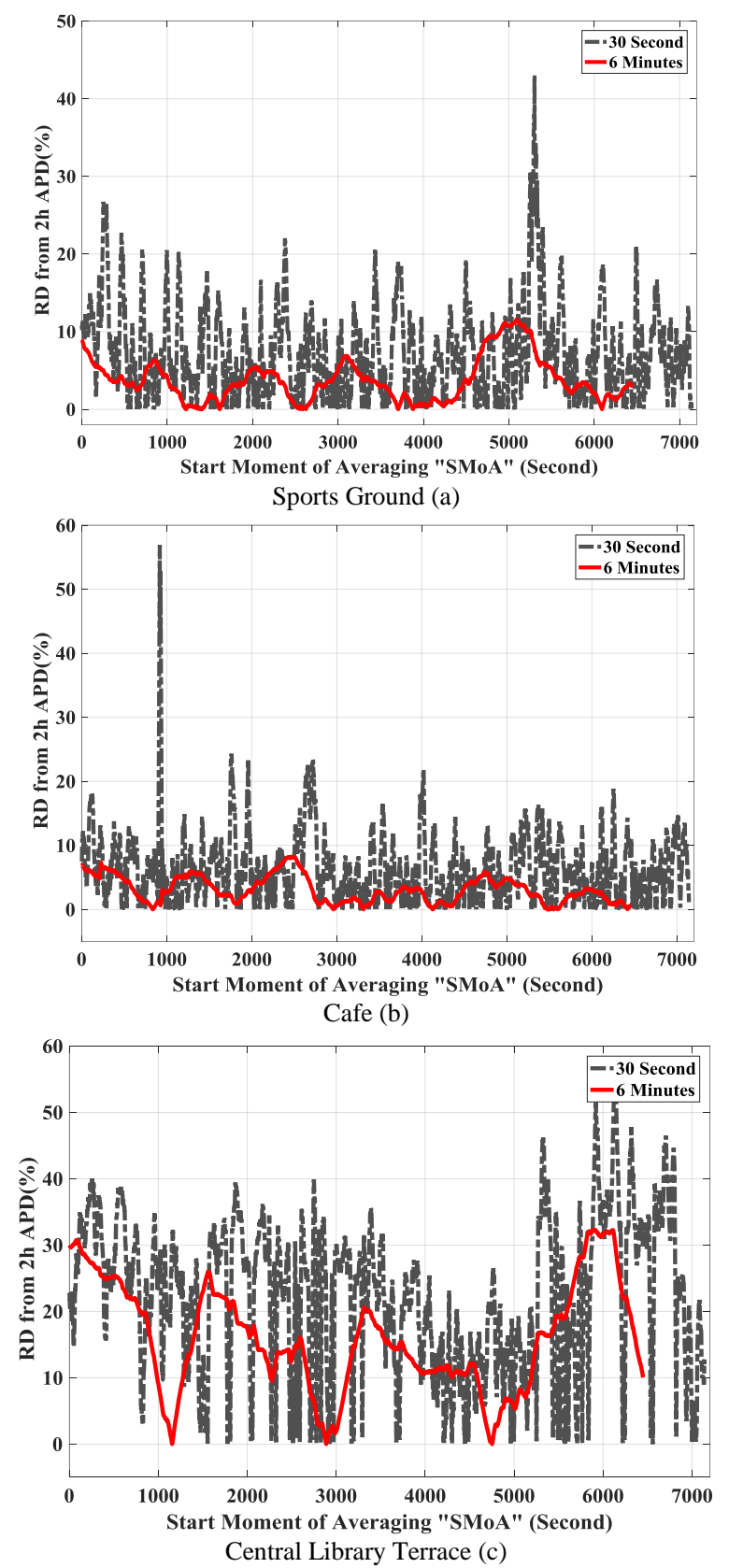

Fig. 8. Measurement of Relative Diversion from the reference APD value for two ATL values, namely 30 seconds and 6 minutes compared for outdoor environments. 


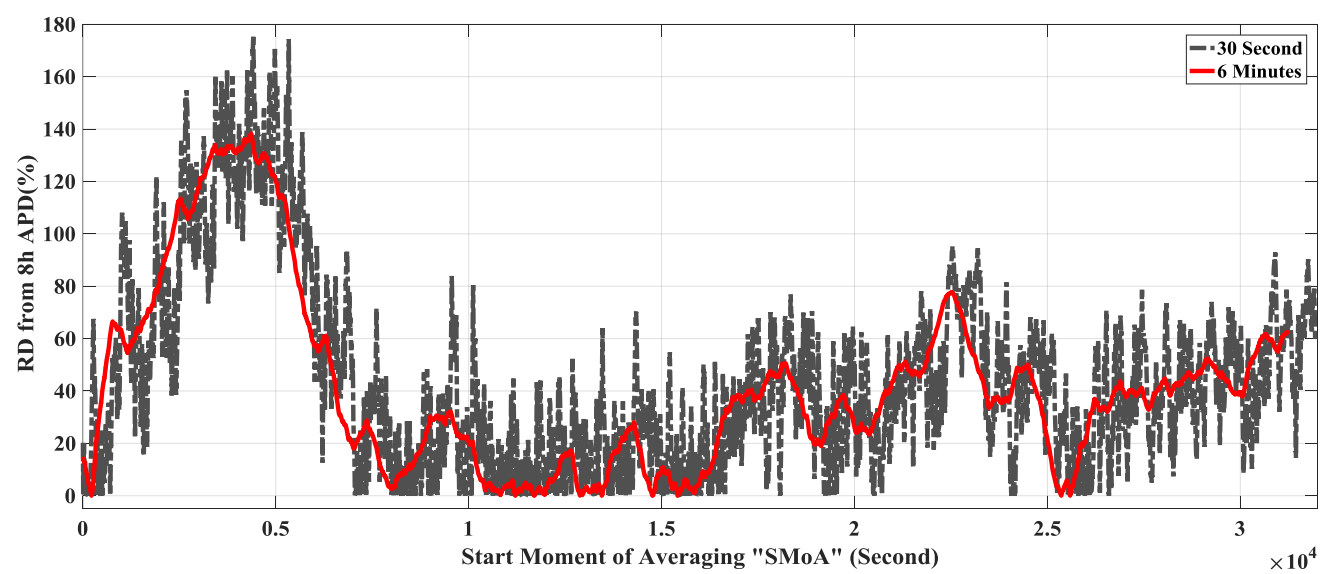

Laboratory $-8 \mathrm{~h}$ in Day-1(a)

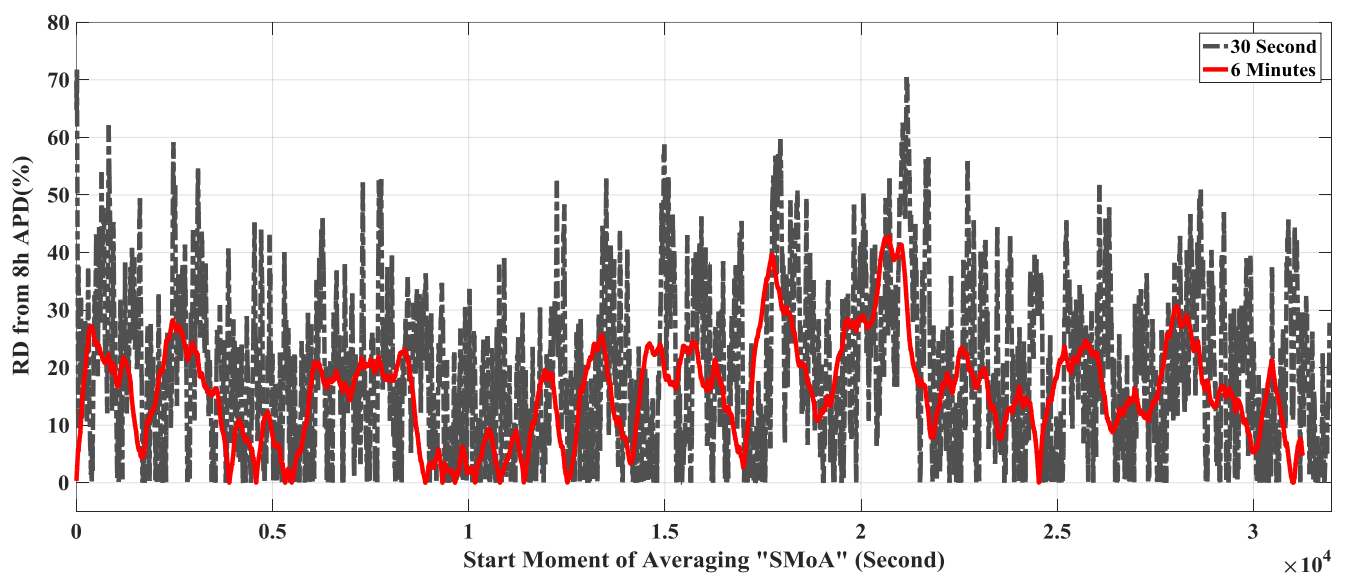

Laboratory $-8 \mathrm{~h}$ in Day-2(b)

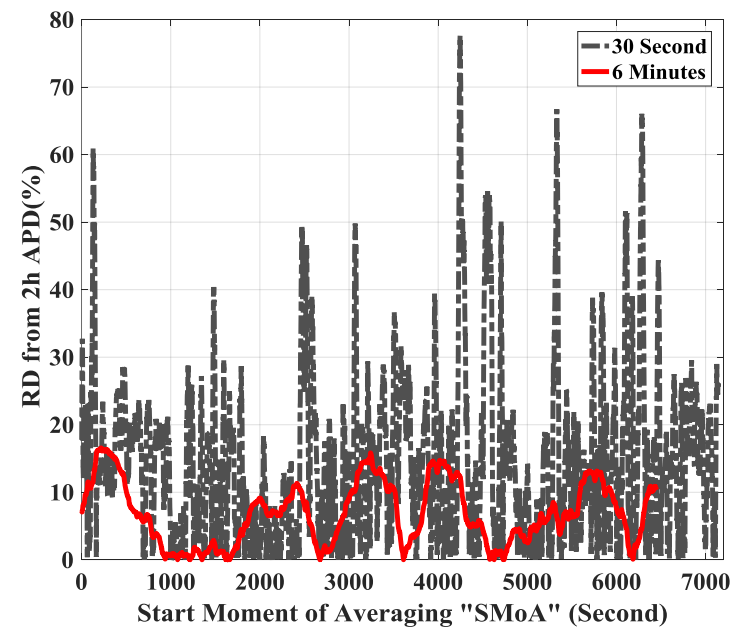

Office $-2 h(b)$

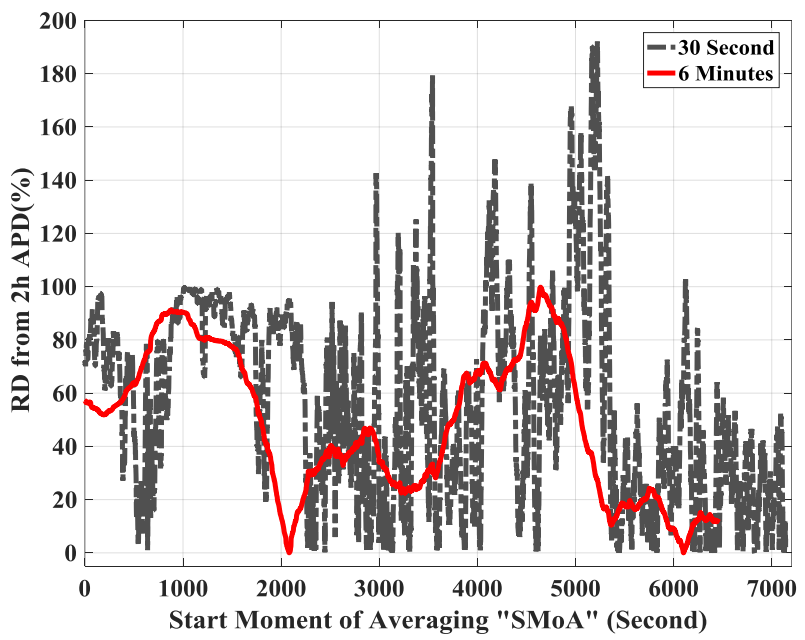

Other Laboratory-2h (c)

Fig. 9. Measurement of Relative Diversion from the refrence APD value for two ATL values, namely 30 seconds and 6 minutes compared for indoor environments. 


\section{E. Benefits of averaging time reduction}

As a result of the above discussion, RF dosimetry can be made at least 10 times faster and therefore cheaper, is less than 6 min averaging is performed.

As an example, a site with 50 test locations requests a measurement. Based on international standards, for each location there is a needs for a spatial averaging on several nearly points, $N$, to determine the value of that location. Based on Canadian standard, these point our determined on a body size plane, for instance a vertical $40 \mathrm{~cm}$ to $70 \mathrm{~cm}$ rectangular plane Assuming $N=9$, this leads to 9 measurements per location, and thus 450 measurements for the whole site. Having 6-minute measurements this leads to 45 hours, or at least a week long mission.

However, by accepting the reasonable reduction of averaging time to 30 seconds, this will reduce to one-day test which not only is cheaper and faster, but also can be afforded by an ordinary people.

The above recommendations are applicable in the environments where there are no abnormal transmitters such as high power radar or noise sources such as industrial facilities. In order to statistically increase the reliability of the results of this work and to generalized its recommendations, more researches and studies are needed.

\section{CONCLUSION}

In this paper, aiming at simplification of RF dosimetry, the results of several general public measurements are studied to show that shorter periods of sampling can be exploited. Based on the statistical analysis of the obtained measurement results, approximately 30 seconds sampling durations can replace well-known but unproven 6-mintue duration for some general public environments. This can reduce the required measurement time significantly resulting in faster and cheaper measurements.

\section{ACKNOWLEDGMENTS}

We would like to thank Dr. Mehrdad Saviz from Amir Kabir University of Technology for his valuable comments on the work. Also we would like to thank Mr. Alireza Pourafzal for his contribution in the measurements and analysis.

\section{REFERENCES}

[1] R. Acharya, D. Kumar, and G. Mathur, "Study of Electromagnetic Radiation Effects on Human Body and Reduction Techniques," in Optical and Wireless Technologies: Springer, 2018, pp. 497505.

[2] A. Lerchl et al., "Tumor promotion by exposure to radiofrequency electromagnetic fields below exposure limits for humans," Biochemical and biophysical research communications, vol. 459, no. 4, pp. 585-590, 2015.

[3] F. de Vocht, "Inferring the 1985-2014 impact of mobile phone use on selected brain cancer subtypes using Bayesian structural time series and synthetic controls," Environment international, vol. 97, pp. 100-107, 2016.
[4] I. Calvente, M. Fernandez, J. Villalba, N. Olea, and M. Nunez, "Exposure to electromagnetic fields (non-ionizing radiation) and its relationship with childhood leukemia: a systematic review," Science of the total environment, vol. 408, no. 16, pp. 3062-3069, 2010.

[5] K. Roser et al., "Personal radiofrequency electromagnetic field exposure measurements in Swiss adolescents," Environment international, vol. 99, pp. 303-314, 2017.

[6] J. J. Oh, S.-S. Byun, S. E. Lee, G. Choe, and S. K. Hong, "Effect of electromagnetic waves from mobile phones on spermatogenesis in the era of 4G-LTE," BioMed research international, vol. 2018, 2018.

[7] N. Pasquino, "Measurement and analysis of human exposure to electromagnetic fields in the GSM band," Measurement, vol. 109, pp. 373-383, 2017.

[8] H. Othman, M. Ammari, M. Sakly, and H. Abdelmelek, "Effects of prenatal exposure to WIFI signal $(2.45 \mathrm{GHz})$ on postnatal development and behavior in rat: influence of maternal restraint," Behavioural brain research, vol. 326, pp. 291-302, 2017.

[9] A. Lowden, R. Nagai, T. Åkerstedt, K. Hansson Mild, and L. Hillert, "Effects of evening exposure to electromagnetic fields emitted by $3 \mathrm{G}$ mobile phones on health and night sleep EEG architecture," Journal of sleep research, p. e12813, 2019.

[10] J. Miyakoshi, H. Tonomura, S. Koyama, E. Narita, and N. Shinohara, "Effects of Exposure to $5.8 \mathrm{GHz}$ Electromagnetic Field on Micronucleus Formation, DNA Strand Breaks, and Heat Shock Protein Expressions in Cells Derived From Human Eye," IEEE transactions on nanobioscience, vol. 18, no. 2, pp. 257-260, 2019.

[11] E. Chiaramello et al., "Radio Frequency Electromagnetic Fields Exposure Assessment in Indoor Environments: A Review," International journal of environmental research and public health, vol. 16, no. 6, p. 955, 2019.

[12] H. Jalilian, M. Eeftens, M. Ziaei, and M. Röösli, "Public exposure to radiofrequency electromagnetic fields in everyday microenvironments: An updated systematic review for Europe," Environmental research, 2019.

[13] M. Ibrani, E. Hamiti, L. Ahma, and B. Shala, "Assessment of personal radio frequency electromagnetic field exposure in specific indoor workplaces and possible worst-case scenarios," AEU-International Journal of Electronics and Communications, vol. 70, no. 6, pp. 808-813, 2016.

[14] R. N. Iyare, V. Volskiy, and G. A. Vandenbosch, "Study of the correlation between outdoor and indoor electromagnetic exposure near cellular base stations in Leuven, Belgium," Environmental research, vol. 168, pp. 428-438, 2019. 
[15] J. C. Lin, "A new IEEE standard for safety levels with respect to human exposure to radiofrequency radiation," IEEE Antennas and Propagation Magazine, vol. 48, no. 1, pp. 157159, 2006.

[16] I. Guideline, "Guidelines for limiting exposure to time-varying electric, magnetic, and electromagnetic fields (up to $300 \mathrm{GHz}$ )," Health phys, vol. 74, no. 4, pp. 494-522, 1998.

[17] K. R. Foster, A. Lozano - Nieto, P. J. Riu, and T. S. Ely, "Heating of tissues by microwaves: A model analysis," Bioelectromagnetics: Journal of the Bioelectromagnetics Society, The Society for Physical Regulation in Biology and Medicine, The European Bioelectromagnetics Association, vol. 19, no. 7, pp. 420-428, 1998.

[18] IEEE Standards Coordinating Committee, "IEEE standard for safety levels with respect to human exposure to radio frequency electromagnetic fields, $3 \mathrm{kHz}$ to $300 \mathrm{GHz}$," IEEE C95. 1-1991, 1992.

[19] A. Papoulis and S. U. Pillai, Probability, random variables, and stochastic processes. Tata McGraw-Hill Education, 2002. 